\title{
Aproximación multimetodológica en el estudio de las representaciones sobre Trabajos Prácticos de Laboratorio
}

\author{
Erica Gabriela Zorrilla (D) \\ Consejo Nacional de Investigaciones Cientificas y Técnicas (CONICET). Argentina. \\ ericagabriela@gmail.com
}

\author{
Claudia Alejandra Mazzitelli \\ Instituto de Investigaciones en Educación en las Ciencias Experimentales (IIECE). Facultad de Filosofía, \\ Humanidades y Artes- Universidad Nacional de San Juan.Argentina.mazzitel@ffha.unsj.edu.ar
}

[Recibido: 9 marzo 2020. Revisado: 29 mayo 2020. Aceptado: 16 junio 2020]

Resumen: Este artículo presenta un estudio sobre los trabajos prácticos de laboratorio (TPL) a la luz de las representaciones sociales (RS). El objetivo de esta investigación es identificar y analizar las RS sobre los TPL de 29 docentes de Ciencias Naturales de la provincia de San Juan, Argentina, utilizando técnicas asociativas e interrogativas, como la escala Likert, la técnica de evocación y jerarquización y las frases incompletas. En este artículo intentamos establecer qué técnicas resultan de mayor utilidad en este tipo de estudios, al permitirnos identificar de forma más confiable las RS y, por ende, resulten predictoras del desempeño docente vinculado a los TPL. Los resultados obtenidos nos permiten señalar la necesidad de una aproximación multimetodológica a la hora de estudiar las RS, dada la complejidad que involucran las representaciones, como así también, la mayor contribución de las técnicas asociativas al estudio de las RS.

Palabras clave: Representaciones Sociales, Trabajos Prácticos de Laboratorio, Ciencias Naturales, Enseñanza, Aprendizaje.

\section{Multimethodological Approach In The Study Of The Representations On Laboratory Practical Works}

Abstract: This article presents a study on practical laboratory work (PLW) considering social representations (SR). The objective of this research is to identify and analyze the SR on the PLW of 29 teachers of Natural Sciences from the province of San Juan, Argentina, using associative and interrogative techniques. Among those used are the Likert scale and the techniques of evocation and hierarchization and incomplete sentences. In this article, we will try to establish which techniques are most useful in this type of studies by allowing us to reliably identify the SR, therefore, being predictive of the teaching performance linked to the PLW. The results obtained in this study will allow us to draw attention to the need for a multimethodological approximation at the time of studying the SRs, given the complexity involving the representations, as well as about the important contribution of associative techniques to the study of SR.

Keywords: Learning, Natural Sciences, Practical laboratory Work, Social Representations, Teaching.

Para citar este artículo: Zorrilla E. G. y Mazzitelli C.A. (2021) Aproximación multimetodológica en el estudio de las representaciones sobre Trabajos Prácticos de Laboratorio. Revista Eureka sobre Enseñanza y Divulgación de las Ciencias 18(2), 2601 doi: 10.25267/Rev_Eureka_ensen_divulg_cienc.2021.v18.i2.2601

\section{Introducción}

Las prácticas de laboratorio constituyen herramientas relevantes para la enseñanza y el aprendizaje de Ciencias Experimentales, a la hora de favorecer la construcción de los conocimientos disciplinares. Sin embargo, en la realidad áulica, puede observarse que estas son un recurso poco aprovechado (Schauble, Glaser, Duschl, Schulze y John, 1995; Merino y Herrero, 2007). Por otra parte, Nieda (1994), analiza varias razones que suelen exponerse relacionadas con la reducción en el tiempo dedicado a la experimentación en las clases de Ciencias Naturales. Es así que algunos autores destacan que existen, a veces, en el profesorado inconsistencias entre lo que se enuncia y lo que se realiza en la práctica (Nieda, 1994; Cabrera Cuevas, 2003; Guirado, 2013). 
Estas contradicciones son un aspecto a considerar en este trabajo, a partir de una profundización de su estudio desde la perspectiva de las Representaciones Sociales (RS), ya que, como considera Rodríguez-Salazar (2011), existen cogniciones que forman parte de las representaciones, pero que no son expresadas por los sujetos en condiciones normales de producción de información, pues estas podrían poner en duda valores o normas apreciadas dentro del grupo social al cual pertenecen. Por esto, el posible desfase entre lo dicho y lo actuado precisa de una lectura interpretativa integral, donde se pueda comprender el sentido del mismo, en qué medida los sujetos son conscientes, entre otras cuestiones.

Moscovici $(1961,1979)$ define a las RS como modalidades particulares de conocimiento, cuya función es la elaboración de los comportamientos y la comunicación entre los individuos. Además, señala que tienen un carácter dinámico y a la vez una estructura estable. Así, al interior de la teoría de las representaciones sociales encontramos dos enfoques o modos de abordar su estudio, un enfoque procesual y un enfoque estructural (Banchs, 2000). Esta autora señala que la versatilidad del concepto de representaciones sociales involucra también diferentes modos de apropiación de la teoría, y en tal sentido es que deben considerarse estos dos enfoques. El enfoque procesual focaliza el estudio de las RS en el aspecto dinámico del proceso de construcción de las mismas -desde una mirada hermenéutica-, mientras que desde el enfoque estructural se pone el énfasis en el estudio de la estructura de las RS, la forma en la que se organiza el contenido de la representación. Esto se ve reflejado en la variedad de modos de investigar las RS, debido a que se trata de un fenómeno complejo, siendo el mismo Moscovici (1988) quien alienta los abordajes multimetodológicos. En tal sentido Jodelet (2011) afirma que: el ámbito de la educación es propicio para ser estudiado “...en términos de RS y sus diversas metodologías" (p. 141) y que las RS pueden detectarse en las prácticas concretas y a través del discurso de los sujetos.

Jodelet (1986) menciona algunas de las características de las RS señalando que constituyen un conocimiento espontáneo, de sentido común, implícito; que se construye a través de las experiencias y el intercambio de conocimiento en la interacción social y es, por lo tanto, un conocimiento socialmente construido y compartido y que tiene una finalidad práctica, ya que permite comprender y explicar nuestra realidad, participando en la construcción social de la misma. Atendiendo a esto último, las representaciones sociales funcionarían como "un sistema de interpretación de la realidad que rige las relaciones de los individuos con su entorno físico y social, ya que determinará sus comportamientos o sus prácticas" (Abric, 2001, p. 13).

Las RS son un abordaje pertinente para analizar la práctica docente en Ciencias Naturales, ya que permiten detectar factores que obstaculizan y otros que benefician la enseñanza y el aprendizaje. Por ejemplo, en un estudio realizado por Mazzitelli y Aparicio (2009), se detectaron actitudes positivas relacionadas con el conocimiento disciplinar y el aprendizaje de las Ciencias Naturales en contrapunto con la presencia de actitudes negativas asociadas a la enseñanza de estas ciencias. Los resultados obtenidos les permitieron concluir que existe un problema relacionado con la enseñanza como proceso más que con el contenido que se enseña, y esto se vincula, entre otras cosas, con una falta de adecuación de los recursos de enseñanza a las necesidades de los estudiantes.

Por otra parte, el quehacer docente influye en la construcción de las RS de sus estudiantes, ya que esta construcción ocurre en función de las prácticas y de los valores de los grupos sociales en los cuales se encuentran inmersos los sujetos. De esta manera, la representación que los estudiantes construyen acerca de aprender y enseñar Ciencias Naturales se encuentra en estrecha relación con el discurso y la práctica de los docentes (Mazzitelli, 2013). Así, es que podemos esperar que las RS puedan observarse en el desempeño docente, ya que existe una vinculación entre estas y los comportamientos.

Atendiendo a que las RS son un conocimiento implícito, es importante favorecer su explicitación ya que de esta manera los sujetos pueden reflexionar sobre su existencia y su influencia en los 
comportamientos, para lograr una comprensión más acabada de los fenómenos sociales que describen y para promover cambios si fueran necesarios.

En este contexto cobran especial importancia las técnicas utilizadas para la indagación de las RS. Alrededor de los estudios realizados bajo la perspectiva de las RS, como ya se mencionó, puede observarse un pluralismo metodológico. Petracci y Kornblit (2007), señalan que los investigadores pertenecientes a este campo se caracterizan por la búsqueda permanente de diseños que permitan un acercamiento apropiado a las RS, así como a su vínculo con las prácticas sociales. Abric (2001), argumenta que una aproximación multimetodológica, es la forma de acceder a los diferentes niveles de análisis presentes en las RS. De esta manera, la información obtenida mediante distintas fuentes permite una comprensión más compleja del objeto de estudio (Castorina y Barreiro, 2012).

Con respecto a la metodología usada para identificar las RS, se encuentran diversas técnicas que pueden resultar de utilidad para ello. Abric (2001) agrupa las técnicas de recolección de datos en dos tipos: las técnicas interrogativas y las técnicas asociativas. Las primeras consisten en recolectar la expresión de los sujetos (verbal o figurativa) en relación con el objeto de la representación. Entre ellas se encuentran las entrevistas, cuestionarios, tablas inductoras, soportes gráficos, dibujos, escalas, entre otras. En cuanto a las técnicas asociativas, se relacionan a expresiones más espontáneas y menos controladas, como es el caso de las técnicas de evocación con asociación libre y jerarquización. Este autor resalta los beneficios del uso de éstas últimas ya que reducen las dificultades o limitaciones de la expresión discursiva, favoreciendo así el carácter proyectivo de la producción (Abric, 2001; Mazzitelli, 2010; Barraza Macías y Méndez Zúñiga, 2013).

Teniendo en cuenta lo presentado anteriormente, realizamos un estudio con docentes de Ciencias Naturales, con el fin de identificar y analizar sus RS sobre los TPL, de modo de realizar una caracterización de los mismos, utilizando distintas técnicas. En este artículo intentamos establecer qué técnicas resultan de mayor utilidad en este tipo de estudios, al permitirnos identificar de forma más confiable las RS y, por ende, resulten predictoras del desempeño docente vinculado a los TPL.

\section{Metodología}

Se trabajó con 29 docentes de nivel secundario de la provincia de San Juan, Argentina. Participaron docentes de Física, Química y Biología, que pertenecen a escuelas de gestión estatal, privada y dependientes de la Universidad Nacional de San Juan. El 56\% de los docentes tiene más de 10 años de antigüedad (docentes expertos), aunque también hay un porcentaje considerable de docentes novatos que recién inician su carrera (44\%).

Tomando en cuenta que es necesario acceder al contenido y la organización de la representación y considerando una aproximación multimetodológica, el instrumento usado en este estudio incluyó las siguientes técnicas:

- Una técnica de evocación y jerarquización: Para este caso, se propuso como término inductor la expresión "prácticas de laboratorio" y se pidió mencionar 5 palabras asociadas a este. Cabe aclarar que, aunque en las investigaciones actuales para referirse a estas actividades se utiliza la expresión "trabajos prácticos de laboratorio", en el instrumento utilizado en este estudio se usa la expresión "prácticas de laboratorio", por ser la denominación de uso más habitual entre los docentes. A continuación, y con el fin de conocer la significación de cada uno de los términos anteriormente mencionados, se les pidió que fundamentaran las razones por las cuales habían elegido esas palabras, para de esta manera conocer las significaciones dadas por los sujetos.

- Una técnica de frases incompletas, en la cual los docentes expresaron cómo deberían ser en el nivel secundario las prácticas de laboratorio, y cómo son las prácticas 
experimentales durante la formación docente. Estas frases fueron construidas con la finalidad de que los sujetos pudieran expresar qué piensan respecto de cada uno de los aspectos propuestos de manera abierta y usando sus propias expresiones, por lo cual se pidió incluir los detalles o las aclaraciones que consideraran convenientes para una mejor comprensión de sus opiniones.

- Una escala Likert, donde los sujetos debían señalar su grado de acuerdo con una serie de afirmaciones respecto de las prácticas de laboratorio en Ciencias Naturales (ver Anexo1). Esta escala fue diseñada e implementada en investigaciones anteriores referidas al mismo objeto de investigación (Zorrilla y Mazzitelli, 2015; Zorrilla, 2019).

Por último, y con la finalidad de enriquecer el análisis, se realizaron cinco observaciones no participantes de clases prácticas de laboratorio, efectuando un registro escrito y grabaciones de los audios de las clases. Respecto de esta técnica, Mancovsky (2011) señala que es un trabajo que va entramando datos y teoría a partir de las interacciones que ocurren durante el desarrollo de una clase. El objetivo de esto es comparar los resultados obtenidos a partir de las técnicas anteriormente descriptas con las acciones docentes, para por un lado analizar de manera conjunta las opiniones expresadas por los docentes con su práctica áulica y por otro lado, identificar cuáles de estas técnicas permiten acceder mejor a las RS.

Cada una de las partes que componen el instrumento utilizado recibió un procesamiento diferente, acorde a las características de las técnicas. A continuación, se presenta la descripción de los procedimientos realizados.

En el caso de la técnica de evocación y jerarquización, una vez concluido el proceso de recolección de las expresiones asociadas al término inductor "prácticas de laboratorio", y dada la gran cantidad de palabras que se presentan en esta técnica al igual que las múltiples significaciones que pueden tomar, se elaboraron categorías a fin de agrupar las expresiones mencionadas. Para construir dichas categorías se consideró además de los términos, la significación otorgada a dichas palabras por los sujetos. Posteriormente, se realizó triangulación con otros investigadores (Cea D’Ancona, 1999; Vasilachis, 1992) a fin de considerar las opiniones de especialistas en el área de enseñanza de las Ciencias Naturales para ajustar la inclusión de las palabras en cada una de las categorías. Las categorías resultantes de este proceso pueden visualizarse en la Tabla 1:

Tabla 1. Categorías de análisis.

\begin{tabular}{|l|l|l|}
\hline Categorías & Descripción & Ejemplos \\
\hline Actitudes y características & $\begin{array}{l}\text { Agrupa las expresiones que señalan } \\
\text { características relacionadas con el } \\
\text { desarrollo de los TPL, así como también } \\
\text { las actitudes asociadas }\end{array}$ & Orden, divertidas, compañerismo. \\
\hline Conocimiento científico & $\begin{array}{l}\text { Incluye palabras que se vinculan con el } \\
\text { ámbito de las Ciencias Naturales y las } \\
\text { prácticas de laboratorio. }\end{array}$ & $\begin{array}{l}\text { Física, membrana plasmática, } \\
\text { sustancia. }\end{array}$ \\
\hline Enseñanza y aprendizaje & $\begin{array}{l}\text { Contiene las palabras relacionadas con los } \\
\text { procesos de enseñanza y de aprendizaje }\end{array}$ & Profesor, práctica, estudio. \\
\hline $\begin{array}{l}\text { Materiales y equipamiento de } \\
\text { laboratorio }\end{array}$ & $\begin{array}{l}\text { Se refiere a los elementos que se utilizan } \\
\text { para el desarrollo de las prácticas de } \\
\text { laboratorio. }\end{array}$ & $\begin{array}{l}\text { Microscopio, tubo de ensayo, } \\
\text { erlenmeyer. }\end{array}$ \\
\hline Procesos y procedimientos & $\begin{array}{l}\text { Incluye palabras que se refieren a los } \\
\text { procedimientos propios de la construcción } \\
\text { del conocimiento científico. }\end{array}$ & $\begin{array}{l}\text { Observar, pasos para realizar un } \\
\text { laboratorio, experimentación, } \\
\text { hipótesis. }\end{array}$ \\
\hline
\end{tabular}


Posteriormente se calculó la frecuencia de aparición de las palabras y el orden de importancia asignado a los elementos mencionados y jerarquizados por los sujetos (Abric, 2003), lo cual permite la identificación de la estructura de la representación (Petracci y Kornblit, 2007; Graça, Moreira y Caballero, 2004; Mazzitelli, 2012).

Siguiendo el proceso realizado por Mazzitelli (2012,2015), el criterio utilizado para decidir cuándo la importancia asignada a cada categoría es alta o baja fue considerar para cada una de ellas los promedios, obtenidos de los valores de importancia asignados a cada una de las palabras que ingresaron a las respectivas categorías (entre 1 y 5). Así, la importancia será alta cuando el valor del promedio para la categoría sea menor que 3 y la importancia será baja cuando el promedio se encuentre entre 3 y 5 .

En relación con la frecuencia de aparición de cada categoría, para decidir cuándo considerarla grande o pequeña, se analizaron las frecuencias de todas las categorías por separado y se calculó la media aritmética (p) entre la mayor y la menor frecuencia de las categorías. Luego, si $\mathrm{n}$ (frecuencia de aparición de una categoría) es mayor o igual a p, la frecuencia es grande y si n (frecuencia de aparición de una categoría) es menor que p, la frecuencia es pequeña.

Se destaca que la frecuencia calculada y la importancia asignada permiten decidir acerca de la centralidad de los términos mencionados. De esta manera, se procedió a agrupar las categorías en cuatro zonas que permiten reconocer la estructura de las RS:

- Núcleo: frecuencia alta-importancia grande

- Primera periferia: frecuencia alta- importancia pequeña

- Segunda periferia: frecuencia baja- importancia pequeña

- Elementos de contraste: frecuencia baja- importancia grande

Una vez identificadas las RS de los docentes participantes, se analizó la estructura de la RS.

En cuanto a la técnica de frases incompletas, para el procesamiento y análisis de las respuestas dadas por docentes, se elaboraron categorías de análisis. Cabe destacar que una misma respuesta puede incluirse en más de una categoría por las diferentes ideas expresadas, ya que se buscó captar toda la riqueza de las opiniones (ver Anexo 2). Además, con el fin de reducir un posible sesgo tanto en la construcción de las categorías como en la agrupación de las respuestas, se realizó triangulación entre investigadores. Así, considerando las opiniones de especialistas en diversas disciplinas relacionadas con la enseñanza de las Ciencias Naturales, se ajustó el análisis para acordar la inclusión de las frases en las diferentes categorías. Una vez determinadas las categorías e incluidas las frases de los docentes, se realizó el cálculo de la frecuencia de las mismas, con el objetivo de presentar estos resultados en una gráfica apropiada que permita visualizar de manera comparada la frecuencia de aparición de cada categoría.

Respecto de la escala Likert, con los datos relevados a través de esta técnica, se construyeron perfiles actitudinales. Para esto, se calculó el promedio de las opciones elegidas para cada una de las afirmaciones y estos resultados fueron volcados en un gráfico, donde en el eje de las abscisas figuran las variables que forman parte de la escala, mientras que los valores del eje de las ordenadas corresponden a las opciones incluidas como posibles respuestas, las cuales varían entre el acuerdo total (1) y el desacuerdo total (5). Cabe destacar que el análisis realizado a partir de esta técnica permite una caracterización general de la muestra.

En lo referido al procesamiento de las observaciones de las clases prácticas de laboratorio, se analizaron los registros escritos y grabados de estas clases teniendo en cuenta la clasificación de niveles de apertura. Para esto, se utilizó una clasificación que surge de la combinación de dos clasificaciones previas (Zorrilla, 2019), la esbozada por Herron (1971) y la presentada por 
Priestley (1997). De esta manera, se asoció a cada nivel de apertura una descripción sintética del tipo de actividades realizadas, los procesos cognitivos requeridos por parte de los estudiantes, la presencia explícita o la ausencia en el protocolo de los objetivos, materiales, métodos y soluciones de dicha práctica, así como también quién es el sujeto que la realiza. Además, a fin de comparar la práctica áulica con lo expresado por los docentes en el instrumento, se utilizó la relación entre el grado de apertura de los TPL desarrollados con el modelo didáctico que sustenta las prácticas (ver Anexo 3).

\section{Análisis de resultados}

A continuación se presentan los resultados obtenidos con cada una de las técnicas.

\section{Técnica de evocación y jerarquización}

A partir del procesamiento explicitado en el apartado anterior -Metodología-, se identificó la estructura de la RS de los docentes, acerca de las prácticas de laboratorio, que se muestra en la Tabla 2. A continuación de la misma se analizará dicha estructura y se mencionarán los ejemplos más destacados en relación con la frecuencia de aparición de las palabras que componen cada categoría.

Tabla 2. Estructura de la RS de docentes acerca de las prácticas de laboratorio.

\begin{tabular}{|l|l|}
\hline Estructura & Categorías \\
\hline Núcleo & Enseñanza y aprendizaje \\
\hline Primera periferia & $\begin{array}{l}\text { Características y actitudes } \\
\text { Procesos y procedimientos }\end{array}$ \\
\hline Segunda periferia & $\begin{array}{l}\text { Conocimiento científico } \\
\text { Materiales y equipamiento de laboratorio }\end{array}$ \\
\hline
\end{tabular}

Como puede observarse, la estructura de las RS que predomina se encuentra centrada en elementos relativos a la enseñanza y el aprendizaje en las prácticas experimentales. Se destaca la mención de procesos relacionados con la construcción del conocimiento como "aprendizaje significativo" o "evaluación formativa".

Por otra parte, la primera periferia de la representación se encuentra compuesta por expresiones relacionadas tanto a procedimientos como a características y actitudes relativas a las prácticas de laboratorio. Es así, que esta zona de la representación presenta un carácter procedimental y actitudinal, asociado a la presencia de expresiones tales como "destreza", "habilidades", "interpretación" o "procedimientos", en cuanto a lo procedimental, y en cuanto a lo actitudinal, "motivación", "perseverancia", "curiosidad", lo cual evidencia una valoración positiva de los TPL. Cabe señalar la presencia (con menor orden de importancia) de palabras que refieren a características negativas como "falta de tiempo", "falta de ayudante" o "desinterés de los alumnos".

Respecto a la ubicación en la segunda periferia de la categoría Conocimiento científico, puede inferirse que si bien no es un aspecto al que los docentes le asignen una gran importancia, consideran que los TPL si bien requieren un bagaje conceptual para poder ser realizados. Entre las palabras que se destacan en esta categoría se encuentran "conocimiento" y "contenidos". En esta parte de la estructura también encontramos la categoría Materiales y equipamiento de laboratorio. En este caso se destacan las palabras "laboratorio", "sustancia" y "materiales". 


\section{Técnica de frases incompletas}

A continuación, en las Figuras 1 y 2, se presenta la distribución de las frecuencias porcentuales para las categorías elaboradas para la técnica de frases incompletas considerando las respuestas dadas por todos los docentes de la muestra. Cabe recordar que una respuesta puede encontrarse incluida en más de una categoría:

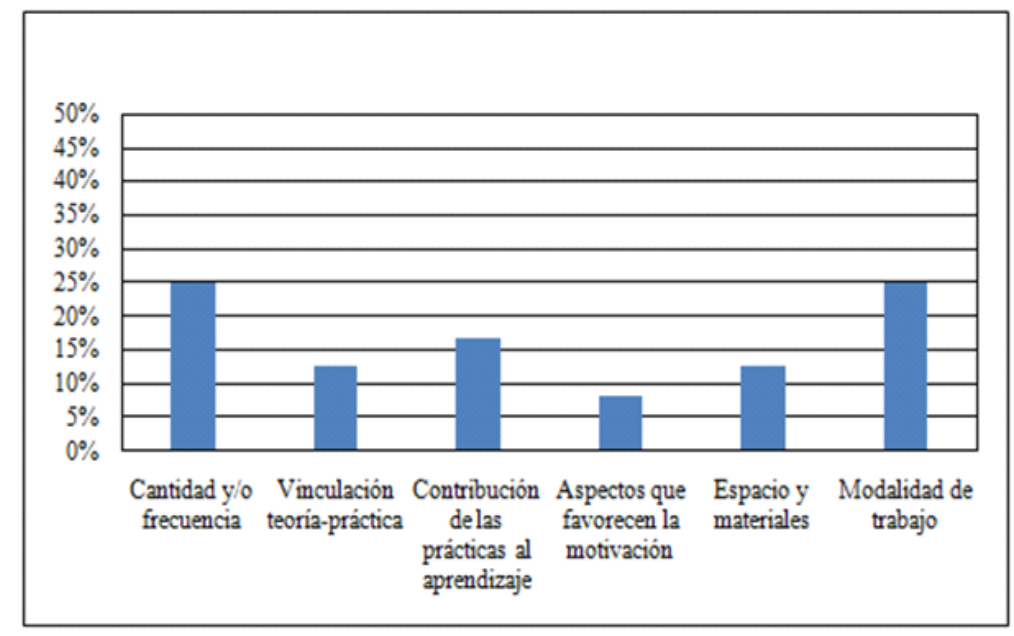

Figura 1. Distribución de frecuencias porcentuales para las categorías del tronco verbal "Las prácticas de laboratorio en la escuela secundaria deberían ser...". Fuente: Elaboración propia.

Como puede observarse en la Figura 1, las categorías que aparecen con mayor frecuencia hacen referencia tanto a la modalidad de trabajo en las prácticas experimentales como a la frecuencia con la cual se realizan. Dentro de las caracterizaciones expresadas respecto a la forma de trabajo en el laboratorio, se menciona mayoritariamente que los TPL se encuentren adaptados a la edad de los alumnos. En cuanto a la categoría Cantidad y/o frecuencia, todas las respuestas muestran una exigencia de mayor cantidad y periodicidad de las prácticas. De este resultado se podría inferir una escasa implementación del trabajo experimental en el nivel secundario, lo cual también es señalado en numerosas investigaciones vinculadas a la enseñanza de las Ciencias Naturales (Hodson, 1994; Daza Pérez y Moreno Cárdenas, 2010; Fernández, 2010; Mengascini y Mordeglia, 2014 ).

Otra categoría que se destaca es Contribución de las prácticas al aprendizaje. En esta categoría, las respuestas de los docentes señalan principalmente que los TPL deberían acompañar la construcción de los aprendizajes en Ciencias Naturales.

Con menor frecuencia se encuentran las respuestas que pertenecen a las categorías relativas a la vinculación entre teoría y práctica, así como al espacio y los materiales con los cuales se realizan las prácticas. En el primer caso, todas las expresiones detallan que debe existir una vinculación entre los TPL realizados y la teoría brindada en las materias, ya que estos permiten la comprensión de diferentes conceptos y procedimientos científicos. Por otra parte, en el segundo caso, se destaca en todas las expresiones la necesidad de material de laboratorio específico para llevar a cabo las prácticas.

Por último, las expresiones que se relacionan con aspectos actitudinales, son las que con menor frecuencia se mencionan. Los docentes que hacen referencia a estos aspectos, consideran a los TPL como herramientas que favorecen la motivación de los estudiantes por el aprendizaje de las Ciencias Naturales.

En resumen, puede destacarse que, excepto aspectos actitudinales, el resto de las categorías tiene una presencia destacada en las opiniones de los docentes. Es así que el amplio espectro de categorías vinculadas a las respuestas podría deberse a que consideran varios factores o 
elementos como importantes o necesarios en las prácticas experimentales, ya que sus respuestas se encuentran vinculadas, en la mayoría de los casos, con más de una categoría.

A continuación, se presenta el análisis de la segunda frase incompleta, "Las prácticas de laboratorio en la formación docente son...":

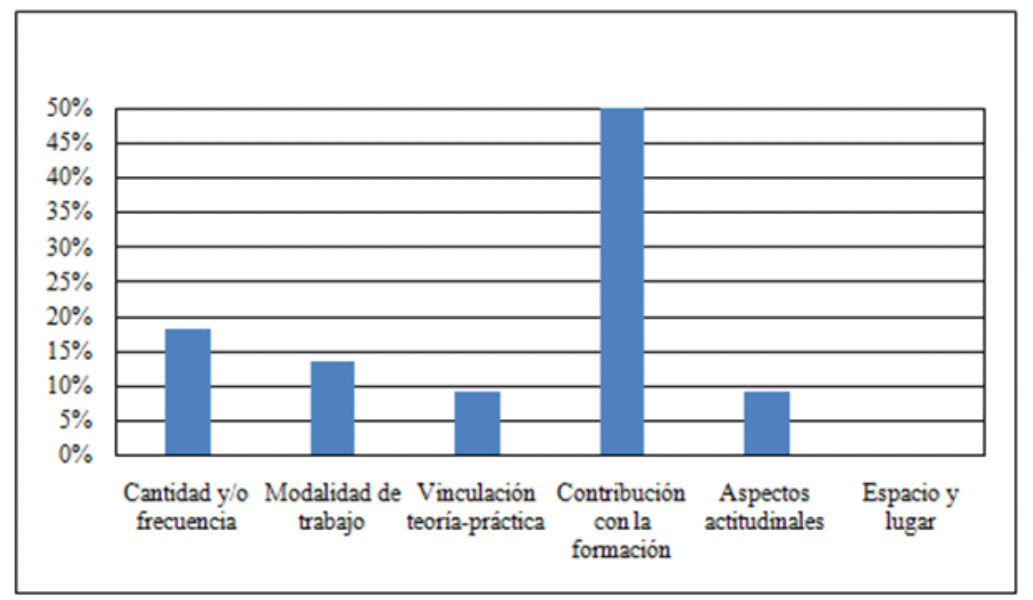

Figura 2. Distribución de frecuencias porcentuales para las categorías del tronco verbal "Las prácticas de laboratorio en la formación docente son...”. Fuente: Elaboración propia.

En la Figura 2 se observa que la categoría que aparece con mayor frecuencia es Contribución de los TPL con la formación docente. Dentro de esta categoría podemos señalar que la mitad de las expresiones brindadas por los docentes manifiestan que los TPL realizados durante la formación docente contribuyen de manera positiva, y la otra mitad los presenta de una manera poco beneficiosa. Esta distribución de respuestas se encuentra relacionada con la antigüedad en la docencia, ya que los docentes novatos expresaron mayor cantidad de opiniones que relacionan los TPL en la formación docente como contribuciones positivas, mientras que los docentes expertos los mencionan como contribuciones poco beneficiosas.

Otra de las categorías que es mencionada en mayor medida es Cantidad y/o frecuencia, donde se incluyeron aquellas expresiones que se refieren no sólo a la cantidad y frecuencia de prácticas realizadas, sino también a la duración de las mismas. En todos los casos para esta categoría, las expresiones hacen referencia a que son escasas tanto la cantidad de prácticas de laboratorio como la duración de las mismas, lo que muestra que al trabajo experimental no se le otorga un lugar de importancia durante la formación docente.

Es importante señalar, que a pesar de las pocas expresiones referidas a la categoría Modalidad de trabajo, todas ellas señalan como característica común de las prácticas en la formación docente el uso de protocolos pautados, que no tienen en cuenta la futura práctica docente. También se encuentran con frecuencia baja las categorías relacionadas con la vinculación entre la teoría y la práctica en los TPL, así como también los aspectos motivacionales relacionados a ellos.

Resulta llamativa la ausencia de respuestas relacionadas con la categoría Espacio y materiales. Esto podría relacionarse con la escasa realización de prácticas experimentales, por lo cual los docentes no logran identificar este aspecto como fortaleza ni como debilidad.

En síntesis, puede destacarse que la polarización existente respecto a si los TPL realizados durante la formación docente contribuyen de manera poco beneficiosa o positiva, se vincula con la antigüedad en la docencia de los profesores de la muestra y podría tener relación con las características de la formación docente inicial en los distintos momentos del sistema educativo. A pesar de esta diferencia, docentes novatos y expertos acordaron de manera unánime acerca 
de la escasa realización de prácticas experimentales durante la formación docente y de que en los casos en los cuales se realizaron TPL, estos en general se desarrollan con protocolos tradicionales, pautados o de bajos niveles de apertura. Es así, que según las opiniones de estos docentes, los TPL en el nivel superior no serían herramientas útiles para la futura práctica docente.

\section{Escala Likert}

A continuación, en la Figura 3 se observa el perfil actitudinal construido a partir de las respuestas dadas por los docentes a la Escala Likert (ver Anexo 1):

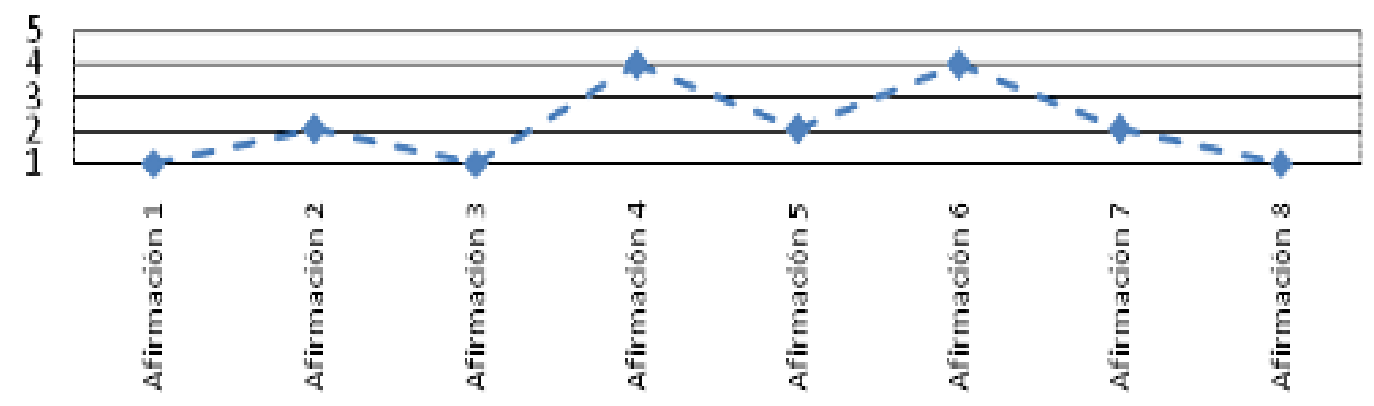

Figura 3. Perfil actitudinal para docentes de nivel secundario. Fuente: Elaboración propia.

Como puede visualizarse en la gráfica, los docentes se muestran muy de acuerdo con respecto a que las prácticas de laboratorio que se realizan durante el dictado de las materias favorecen el aprendizaje de su disciplina (afirmación 1) y que les gusta realizar prácticas experimentales con sus alumnos (afirmación 8). Estas opiniones, resultan un componente muy positivo, ya que además de contribuir con el aprendizaje, la motivación de los docentes propiciaría el desarrollo de un buen clima en el aula. Además, estos docentes también se muestran muy de acuerdo con que las prácticas de laboratorio son presentadas como una actividad reflexiva (afirmación 3), lo cual permitiría inferir que sus propuestas experimentales presentan mayores niveles de apertura que los protocolos tradicionales o de tipo "receta".

Para la afirmación 2 (es necesario modificar la modalidad de trabajo en el laboratorio) y 5 (los TPL pueden ser considerados como investigaciones orientadas), los docentes manifiestan acuerdo, aunque en diferentes niveles. Por otra parte, si bien para la afirmación 7 (las escuelas no ofrecen las condiciones para realizar prácticas de laboratorio) la opinión promedio del grupo se encuentra cercana al acuerdo, en realidad existe una polarización, en la que pueden diferenciarse dos grupos de opiniones: aquellos docentes que se manifiestan de acuerdo, y por otro lado, los que se encuentran indecisos. Esta polarización puede deberse a la diferencia en las opiniones entre docentes expertos y novatos, ya que los primeros manifiestan opiniones entre la indecisión y el desacuerdo, mientras que los segundos se muestran mayoritariamente de acuerdo con lo expresado.

Por último, los docentes expresan su desacuerdo para la afirmación 4 (los TPL pueden ser reemplazados por otras actividades). Esto podría dar cuenta de la percepción de estos docentes acerca de la importancia para el aprendizaje de la realización de actividades experimentales, lo cual debería verse reflejado en la cantidad de TPL que se realizan en las materias de ciencias experimentales. Además, estos docentes también se muestran en desacuerdo con la afirmación 6 (el trabajo en el laboratorio requiere sólo el manejo de contenidos procedimentales), reforzando la idea de que sus propuestas experimentales presentarían mayores niveles de apertura que los protocolos tradicionales.

Al considerar el perfil actitudinal en su conjunto se puede identificar una aparente contradicción entre las opiniones de los docentes. Así, por un lado puede destacarse que los 
docentes opinan que los TPL que se realizan en la escuela durante el cursado de las materias favorecen el aprendizaje de su disciplina y que además les gusta realizar prácticas experimentales con sus estudiantes. Por otro lado, a pesar de esto, los docentes acuerdan con que es necesario modificar la modalidad de trabajo en el laboratorio, lo cual en una primera aproximación podría resultar contradictorio con lo expresado anteriormente.

Al relacionar los resultados obtenidos en los perfiles actitudinales y la producción de frases incompletas, surge la respuesta a por qué destacando tantas características positivas en relación con la realización de los TPL, los docentes consideraban necesaria una modificación del trabajo de laboratorio. La escasa realización de prácticas experimentales en el nivel secundario y en la formación docente inicial y el cuestionamiento hacia la modalidad de trabajo tanto en el nivel secundario como en la formación docente, se establecen entonces como dos causas para la aparente contradicción.

\section{Análisis de observaciones de clases}

A continuación, en la Tabla 3, se presenta el análisis realizado para las observaciones de clases experimentales de cinco docentes, identificando el nivel de apertura al que pertenece el protocolo propuesto para las mismas:

Tabla 3. Análisis de observaciones de TPL en el nivel secundario.

\begin{tabular}{|c|c|c|c|c|c|c|c|}
\hline Clase & $\begin{array}{l}\text { Procesos } \\
\text { cognitivos } \\
\text { requeridos }\end{array}$ & Objetivo & Material & Método & Solución & $\begin{array}{l}\text { Realización } \\
\text { de la } \\
\text { práctica }\end{array}$ & $\begin{array}{l}\text { Nivel de } \\
\text { apertura }\end{array}$ \\
\hline $\begin{array}{l}\text { 1- TPL de } \\
\text { Química (el } \\
\text { agua) }\end{array}$ & $\begin{array}{l}\text { Conocimiento, } \\
\text { comprensión, } \\
\text { aplicación y } \\
\text { análisis }\end{array}$ & Dado & Dado & $\begin{array}{l}\text { Dado en } \\
\text { parte }\end{array}$ & Abierta & $\begin{array}{l}\text { Alumnos- } \\
\text { Docente }\end{array}$ & 6- Abierto \\
\hline $\begin{array}{l}\text { 2- TPL de } \\
\text { Química } \\
\text { (propiedades } \\
\text { intensivas de la } \\
\text { materia) }\end{array}$ & $\begin{array}{l}\text { Conocimiento, } \\
\text { comprensión y } \\
\text { aplicación }\end{array}$ & Dado & Dado & Dado & Abierta & $\begin{array}{l}\text { Alumnos- } \\
\text { Docente }\end{array}$ & $\begin{array}{l}\text { 4- } \\
\text { Entreabierto }\end{array}$ \\
\hline $\begin{array}{l}\text { 3- TPL de } \\
\text { Química } \\
\text { (reacciones } \\
\text { químicas) }\end{array}$ & $\begin{array}{l}\text { Conocimiento, } \\
\text { comprensión y } \\
\text { aplicación }\end{array}$ & Dado & Dado & Dado & Abierta & $\begin{array}{l}\text { Alumnos- } \\
\text { Docente }\end{array}$ & $\begin{array}{l}\text { 4- } \\
\text { Entreabierto }\end{array}$ \\
\hline $\begin{array}{l}\text { 4- TPL de } \\
\text { Física } \\
\text { (movimiento } \\
\text { rectilíneo } \\
\text { uniforme) }\end{array}$ & $\begin{array}{l}\text { Conocimiento y } \\
\text { comprensión }\end{array}$ & Dado & Dado & $\begin{array}{l}\text { Dado en } \\
\text { parte }\end{array}$ & Abierta & $\begin{array}{l}\text { Alumnos- } \\
\text { Docente }\end{array}$ & $\begin{array}{l}\text { 4- } \\
\text { Entreabierto }\end{array}$ \\
\hline $\begin{array}{l}\text { 5- TPL de } \\
\text { Física } \\
\text { (movimiento } \\
\text { rectilíneo } \\
\text { uniforme) }\end{array}$ & $\begin{array}{l}\text { Conocimiento y } \\
\text { comprensión }\end{array}$ & Dado & Dado & Dado & Abierta & $\begin{array}{l}\text { Alumnos- } \\
\text { Docente }\end{array}$ & $\begin{array}{l}\text { 4- } \\
\text { Entreabierto }\end{array}$ \\
\hline
\end{tabular}

Como puede observarse en la Tabla 3, en las clases experimentales observadas hay un predominio de prácticas de laboratorio entreabiertas, correspondientes a un nivel de apertura 4. 
A partir de lo observado, se destaca, independientemente de la disciplina, que:

- Predominan las prácticas experimentales entreabiertas, donde la realización de las mismas está centrada en los estudiantes, pero se encuentran pautados por los docentes los objetivos, métodos y materiales.

- Se prioriza la obtención de datos cuantitativos para luego representarlos en tablas o gráficos, dejando poco lugar para los análisis cualitativos acerca de los fenómenos en estudio y para la transferencia al análisis de situaciones en la vida cotidiana.

- La función principal del docente es a modo de guía, controlando el desarrollo de los procedimientos. En la mayoría de los casos observados, en la realización de la práctica estuvo presente más de un docente (generalmente el profesor responsable del curso y un ayudante de clases prácticas).

- En el caso de los estudiantes, parecerían estar acostumbrados a la realización de prácticas experimentales, ya que conocían en su mayoría la distribución de los elementos en el laboratorio y, además, evidenciaban cierto manejo de los elementos.

Por último, al analizar el modelo didáctico que subyace en este tipo de prácticas, se destacan las siguientes cuestiones:

- En cuanto a los roles (docente/alumno), este tipo de prácticas observadas se corresponden con el modelo por descubrimiento, ya que el docente actúa como guía o coordinador de las actividades experimentales, mientras que los estudiantes cumplen un papel participativo realizando las actividades preferentemente de manera grupal.

- En cuanto a las actividades experimentales, también se corresponden con el modelo anteriormente mencionado, debido a que propician situaciones experimentales que ayudan a los estudiantes a desarrollar habilidades relacionadas con la investigación (es decir, se priorizan las cuestiones procedimentales).

- En relación con las concepciones de ciencia, enseñanza y aprendizaje, presentan características a medio camino entre el modelo por descubrimiento y el constructivista. Esto surge de que el discurso de estos docentes tiene matices constructivistas, aunque en la implementación, sus prácticas son más cercanas al modelo por descubrimiento.

Los resultados encontrados son similares a los identificados en otras investigaciones desde la perspectiva del Conocimiento Pedagógico del Contenido (PCK) y del Conocimiento Didáctico del Contenido (CDK), en las que se ha identificado, un énfasis en los aspectos procedimentales, donde el rol de los estudiantes se limita a la manipulación del instrumental seleccionado para la actividad, reproduciendo los procedimientos propuestos (Sanchez, Odetti y Lorenzo, 2017). Al respecto Fernández Marchesi (2019), afirma que debido a que las concepciones de los docentes se vinculan con las de sus estudiantes, es necesario implementar estrategias que permitan hacer explícitas las concepciones del profesorado, a fin de poder generar cambios genuinos en las prácticas experimentales.

\section{Conclusiones}

En el desarrollo de esta investigación se identificaron y analizaron las estructuras y el contenido de las RS, de modo de obtener una comprensión más integral de las mismas.

A partir de las técnicas asociativas se identificaron RS cuya significación se encuentra centrada en elementos relativos a la enseñanza y el aprendizaje en el laboratorio, con vinculaciones relacionadas a cuestiones actitudinales y procedimentales, aunque desvinculada de los conocimientos científicos. Por otra parte, al considerar los resultados obtenidos para la escala Likert -técnica interrogativa-, se observa que en todos los casos predomina una actitud 
positiva en relación con las prácticas experimentales, poniendo en primer plano su importancia. Hasta este punto, los resultados obtenidos con ambos tipos de técnicas son complementarios.

$\mathrm{Al}$ analizar de forma conjunta las RS identificadas con ayuda de las técnicas asociativas, y las observaciones realizadas en clases de prácticas experimentales, puede destacarse que hay una relación entre los elementos que conforman las RS y las características de estas prácticas. Así, el núcleo de la RS compuesto por la categoría Enseñanza y aprendizaje se relaciona con el lugar brindado por estos docentes a los TPL en sus prácticas, ya que los consideran como un recurso favorecedor de la construcción del conocimiento de Ciencias Naturales en sus alumnos. También podría relacionarse con la presencia de la categoría Procesos y procedimientos en las cercanías del núcleo de la RS, el hecho de que los docentes cumplan el rol de guía, controlando primordialmente el desarrollo procedimental llevado a cabo por sus estudiantes, y que además, en los protocolos estén pautados los elementos procedimentales como objetivos, materiales y métodos. Por otra parte, la presencia de la categoría Conocimiento científico en la zona más alejada al núcleo, también se refleja en los TPL, siendo escasas las actividades que favorecen la vinculación entre la teoría (conocimiento conceptual específico) y la práctica, por ejemplo, al no favorecer la interpretación de los datos a la luz de la teoría, ni la vinculación con situaciones cotidianas.

Profundizando el análisis de la escala Likert en relación con las observaciones de clases prácticas, las opiniones expresadas por los docentes son cercanas a modelos constructivistas, aunque el análisis de sus prácticas y de la estructura de sus representaciones acerca de los TPL presenta características más cercanas al modelo por descubrimiento. Algunos autores como Flores, Caballero Sahelices y Moreira (2009) destacan que el modelo por descubrimiento no es considerado como un enfoque alternativo al tradicional, ya que estos autores señalan que no brinda una solución didáctica adecuada en el caso de las prácticas experimentales.

Llegado este punto, cabe destacar que las diferencias encontradas entre los resultados de las técnicas asociativas y las interrogativas estarían relacionadas con que para las técnicas que son menos espontáneas y que demandan menor producción por parte de los sujetos (escala Likert), las respuestas no evidencian tanto la RS de los docentes, sino una representación idealizada de lo que "debería ser", en este caso, el trabajo en el laboratorio escolar. Sin embargo, las técnicas que son más espontáneas y que permiten una mayor producción por parte de los participantes (frases incompletas y técnica de evocación y jerarquización), permiten una mejor explicitación de sus RS.

Finalmente, considerando estos resultados, se hacen evidentes dos cuestiones, por un lado, lo que expresa Pereira de Sá (1998) al resaltar la necesidad de una aproximación multimetodológica a la hora de estudiar las RS, dada la complejidad que involucran las representaciones y, por otro lado, la mayor contribución de las técnicas asociativas al estudio de las RS.

\section{Referencias}

Abric J.C. (2001) Prácticas sociales y representaciones. México: Ed. Coyoacán.

Abric, J.C. (2003). L'analysestructurale des representations. En: Moscovici, S. (ed.). Méthodelogie des sciences sociales. París: PUF.

Banchs, M. A. (2000). Aproximaciones procesuales y estructurales al estudio de las representaciones sociales. Papers on Social Representation. Threads of discussion, 8, 1-15. 
Barraza Macías A., Méndez Zúñiga A. (2013) Representaciones sociales sobre el estrés laboral en una muestra de docentes de educación primaria. Praxis Investigativa ReDIE: revista electrónica de la Red Durango de Investigadores Educativos, 5(8), 24-33.

Cabrera Cuevas J. D. (2003) Discurso docente en el aula. Estudios pedagógicos, (29), 7-26.

Castorina, J. A., Barreiro, A. (2012). Los usos de las representaciones sociales en la investigación educativa. Educación, Lenguaje y Sociedad, 9(9).

Cea D’Ancona, M.A. (1999). Metodologia Cuantitativa. Estrategias y técnicas de investigación social. Madrid: Ed. Síntesis.

Daza-Pérez, E., Moreno-Cárdenas, J. A. (2010). El pensamiento del profesor deficiencias en ejercicio. Concepciones sobre la enseñanza y el aprendizaje de las ciencias naturales. Revista Electrónica de Enseñanza de las Ciencias, 9(3), 549-568.

Fernandez, N. (coord). (2010). Algo más que locos experimentos para hacer en clases. Manual de trabajos de laboratorio. Argentina: Editorial Utopias.

Fernández Marchesi, N. E. (2019). El conocimiento didáctico del contenido sobre las actividades prácticas de laboratorio por indagación de profesores de Biología. Tesis doctoral. Universidad de Extremadura.

Flores J., Caballero Sahelices M. C., Moreira M. A. (2009) El laboratorio en la enseñanza de las ciencias: Una visión integral en este complejo ambiente de aprendizaje. Revista de investigación, 33(68), 75-111.

Guirado A.M. (2013) Los Modelos Didácticos de docentes de Ciencias Naturales de nivel secundario: reconstrucción a partir de sus concepciones y sus prácticas áulicas. Tesis de doctorado. Universidad Nacional de Cuyo, Mendoza, Argentina.

Guirado A., Mazzitelli C., Olivera A. (2013) Representaciones sociales y práctica docente: una experiencia con profesores de Física y de Química. Revista de Orientación Educacional, 27 (51), 87-105.

Herron M. D. (1971) The nature of scientific enquiry. The School Review, 79(2), 171-212.

Hodson, D. (1994). Hacia un enfoque más crítico del trabajo de laboratorio. Enseñanza de las ciencias. 12 (3), 299-313.

Jodelet, D. (1986). La Representación social: fenómenos, concepto y teoría. En: Moscovici, S. (comp). Psicología social, II. Barcelona: Ed. Paidós.

Jodelet, D. (2011). Aportes del enfoque de las representaciones sociales al campo de la educación. Espacios en blanco. Serie indagaciones, 21(1), 133-154.

Mancovsky V. (2011) La palabra del maestro. Evaluación informal en la interacción de la clase. Buenos Aires, Argentina: Ed. Paidós.

Marcovah I. (2003) La presentación de las representaciones sociales: diálogo con Serge Moscovici. En: Castorina J. A. (comp). Representaciones sociales. Problemas teóricos y conocimientos infantiles. Barcelona: Editorial Gedisa.

Mazzitelli C., Aparicio M. (2009) Las actitudes de los alumnos hacia las Ciencias Naturales, en el marco de las representaciones sociales, y su influencia en el aprendizaje. Revista Electrónica de Enseñanza de las Ciencias, 8 (1).

Mazzitelli C. A. (2012). Representaciones acerca de la enseñanza y el aprendizaje de las ciencias durante la formación docente inicial. Profesorado: Revista de curriculum y formación del profesorado, 16(3), 392-406. 
Mazzitelli C. (2015). La enseñanza de las ciencias y la formación docente inicial: estudio de las representaciones sociales de estudiantes y docentes formadores. San Juan, Argentina: Editorial FFHA - UNSJ.

Mazzitelli C., Aparicio M. (2010) El abordaje del conocimiento cotidiano desde la teoría de las representaciones sociales. Revista Eureka sobre Enseñanz̧a y divulgación de las Ciencias, 7(3), pp. 636-652.

Mengascini, A. S., Mordeglia, C. (2014). Caracterización de prácticas experimentales en la escuela a partir del discurso de docentes de primaria y secundaria. Enseñanza de las Ciencias, 32.

Merino J. M., Herrero F. (2007) Resolución de problemas experimentales de Química: una alternativa a las prácticas tradicionales. Revista Electrónica de Enseñanza de las Ciencias. 6(3), 630-648.

Moscovici, S. (1961). La psychanalyse, son image et son public: étude sur la représentation sociale de la psychanalyse. París: Presses universitaires de France.

Moscovici, S. (1979). El Psicoanálisis, su imagen y su público. Bs. As.: Huemul.

Moscovici, S. (1988). Notes towards a description of Social Representations. European Journal of Social Psychology, 18, 211-250.

Nieda J. (1994) Algunas minucias sobre los trabajos prácticos en la enseñanza secundaria. Alambique: Didáctica de las Ciencias Experimentales, 1(2), 15-20.

Petracci M., Kornblit A. (2007) Representaciones sociales: una teoría metodológicamente pluralista. En Kornblit A. (comp.). Metodologias cualitativas en Ciencias Sociales. (pp. 91 111) Buenos Aires: Editorial Biblos.

Priestley W. (1997) The impact of longer term intervention on reforming physical science teachers' approaches to laboratory instruction: seeking a more effective role for laboratory in science education. En Jiménez Valverde G., Llobera Jiménez R., LlitjósViza A. (2006). La atención a la diversidad en las prácticas de laboratorio de Química: los niveles de abertura. Revista Electrónica de Enseñanza de las Ciencias. 24 (1), pp. 59-70

Rodríguez Salazar T. (2003) El debate de las representaciones sociales en la psicología social. Relaciones. Estudios de historia y sociedad, 24(93).

Sánchez, G. H., Odetti, H. S., Lorenzo, M. G. (2017). La práctica docente en el laboratorio universitario y el conocimiento didáctico del contenido de química inorgánica. Enseñanza de las ciencias: revista de investigación y experiencias didácticas, (Extra), 183-190.

Schauble L., Glaser R., Duschl R.A., Schulze S., J. John. (1995) Students' understanding of the objectives and procedures of experimentation in the science classroom. The Journal of the Learning Sciences, 4 (2), 131-166.

Vasilachis, I. (1992). Métodos cualitativos I. Los problemas teórico-epistemológicos. Buenos Aires, Argentina: Centro Editor de América Latina.

Zorrilla, E., Mazzitelli, C. (2015). Las actitudes de futuros docentes hacia las prácticas de laboratorio en su formación inicial. Tercer Encuentro de Jóvenes Investigadores. Argentina: Universidad Nacional de San Juan.

Zorrilla, E. (2019). Las prácticas de laboratorio en la enseñanza y el aprendizaje de las Ciencias Naturales desde una perspectiva psicosocial. Tesis de Doctorado. Universidad Nacional de Cuyo, Mendoza-Argentina. 
Anexo 1. Escala Likert.

\begin{tabular}{|c|c|c|c|c|c|}
\hline & $\begin{array}{l}\text { Muy de } \\
\text { acuerdo }\end{array}$ & De acuerdo & Indeciso & En desacuerdo & En total desacuerdo \\
\hline $\begin{array}{l}\text { Las prácticas de laboratorio } \\
\text { que se realizan en la escuela } \\
\text { durante el cursado de las } \\
\text { materias favorecen el } \\
\text { aprendizaje de } \\
\text { Química/Física/Biología }\end{array}$ & & & & & \\
\hline $\begin{array}{l}\text { Es necesario modificar la } \\
\text { modalidad de trabajo en el } \\
\text { laboratorio }\end{array}$ & & & & & \\
\hline $\begin{array}{l}\text { Las prácticas de laboratorio } \\
\text { son una actividad reflexiva }\end{array}$ & & & & & \\
\hline $\begin{array}{l}\text { Los prácticos de laboratorio } \\
\text { pueden ser reemplazados por } \\
\text { otras actividades }\end{array}$ & & & & & \\
\hline $\begin{array}{l}\text { Las prácticas de laboratorio } \\
\text { pueden ser } \begin{array}{r}\text { consideradas } \\
\text { como }\end{array} \\
\text { investigaciones }\end{array}$ & & & & & \\
\hline $\begin{array}{l}\text { El trabajo en el laboratorio } \\
\text { requiere sólo el manejo de } \\
\text { contenidos procedimentales }\end{array}$ & & & & & \\
\hline $\begin{array}{l}\text { Las escuelas no ofrecen las } \\
\text { condiciones para realizar } \\
\text { prácticas de laboratorio }\end{array}$ & & & & & \\
\hline $\begin{array}{lcr}\text { Me gusta } & \text { realizar con mis } \\
\text { alumnos } & \text { prácticas } & \text { de } \\
\text { laboratorio } & & \end{array}$ & & & & & \\
\hline
\end{tabular}


Anexo 2. Categorías para la Técnica de Frases Incompletas.

\begin{tabular}{|l|l|}
\hline Categorías & Descripción \\
\hline $\begin{array}{l}\text { Contribución de las prácticas al aprendizaje/la } \\
\text { Contribución de las prácticas a la formación }\end{array}$ & $\begin{array}{l}\text { Incluimos aquellas expresiones que mencionan a las } \\
\text { prácticas de laboratorio en relación con situaciones de } \\
\text { aprendizaje. }\end{array}$ \\
\hline Caracterización de la modalidad de trabajo & $\begin{array}{l}\text { Concentramos las expresiones que mencionan a las } \\
\text { prácticas de laboratorio como una parte importante de } \\
\text { la formación de docentes de Ciencias Naturales. }\end{array}$ \\
\hline Cantidad y/o frecuencia & $\begin{array}{l}\text { Agrupamos las expresiones relacionadas con la forma } \\
\text { de trabajar en las prácticas de laboratorio, teniendo en } \\
\text { cuenta la cantidad de personas, la finalidad de la } \\
\text { práctica, los diferentes momentos de trabajo } \\
\text { prelaboratorio, laboratorio y poslaboratorio), las } \\
\text { normas de seguridad, entre otras características. }\end{array}$ \\
\hline Espacio y materiales & $\begin{array}{l}\text { Agrupamos expresiones que hacen referencia a la } \\
\text { cantidad y/o frecuencia de prácticas realizadas. }\end{array}$ \\
\hline Aspectos actitudinales & $\begin{array}{l}\text { Incluimos las opiniones que hacen referencia al espacio } \\
\text { donde se realizan las prácticas ya los materiales de } \\
\text { laboratorio, como una parte en el proceso de } \\
\text { construcción o desarrollo de las prácticas. }\end{array}$ \\
\hline $\begin{array}{l}\text { Concentramos las expresiones referidas a las } \\
\text { características que los alumnos le atribuyen a las } \\
\text { prácticas de laboratorio y que genera diferentes tipos } \\
\text { de motivación para el aprendizaje. }\end{array}$ \\
\hline $\begin{array}{l}\text { Agrupamos frases relacionadas con el tipo de } \\
\text { vinculación entre teoría y prácticas de laboratorio. }\end{array}$ \\
\hline
\end{tabular}


Anexo 3. Relación entre niveles de apertura y modelos didácticos. Elaboración propia, basada en la propuesta de Herron (1971) y Priestley (1997).

\begin{tabular}{|c|c|c|c|c|c|c|}
\hline Nivel & Objetivo & Material & Método & Solución & $\begin{array}{l}\text { Realización de la } \\
\text { práctica }\end{array}$ & $\begin{array}{l}\text { Modelo didáctico al } \\
\text { que refiere }\end{array}$ \\
\hline 0 & Dado & Dado & Dado & Dada & Docente & Tradicional \\
\hline 1 & Dado & Dado & Dado & Dada & $\begin{array}{l}\text { Docente- } \\
\text { Alumnos }\end{array}$ & Tradicional \\
\hline 2 & Dado & Dado & Dado & $\begin{array}{lr}\text { Dada } & \text { en } \\
\text { parte } & \text { O } \\
\text { abierta } & \end{array}$ & $\begin{array}{l}\text { Docente- } \\
\text { Alumnos }\end{array}$ & Tradicional \\
\hline 3 & Dado & Dado & Dado & Abierta & $\begin{array}{l}\text { Docente- } \\
\text { Alumnos }\end{array}$ & $\begin{array}{l}\text { Transición entre el } \\
\text { modelo tradicional y } \\
\text { el modelo por } \\
\text { descubrimiento }\end{array}$ \\
\hline 4 & Dado & $\begin{array}{l}\text { Dado } \\
\text { todo o en } \\
\text { parte }\end{array}$ & Dado & Abierta & $\begin{array}{l}\text { Alumnos- } \\
\text { Docente }\end{array}$ & Por descubrimiento \\
\hline 5 & Dado & $\begin{array}{l}\text { Dado } \\
\text { todo o en } \\
\text { parte }\end{array}$ & $\begin{array}{l}\text { Dado todo } \\
\text { o en parte }\end{array}$ & Abierta & $\begin{array}{l}\text { Alumnos- } \\
\text { Docente }\end{array}$ & $\begin{array}{l}\text { Transición entre el } \\
\text { modelo por } \\
\begin{array}{l}\text { descubrimiento y el } \\
\text { constructivista }\end{array}\end{array}$ \\
\hline 6 & Dado & Abierto & Abierto & Abierta & Alumnos & Constructivista \\
\hline 7 & $\begin{array}{lr}\text { Dado en } \\
\text { parte } & \text { o } \\
\text { abierto } & \end{array}$ & Abierto & Abierto & Abierta & Alumnos & Constructivista \\
\hline
\end{tabular}

